\title{
Obscuring and Feeding Supermassive Black Holes with Evolving Nuclear Star Clusters
}

\author{
M. Schartmann ${ }^{1,2}$, A. Burkert ${ }^{1,2,3}$, M. Krause ${ }^{1,2}$, M. Camenzind ${ }^{4}$, \\ K. Meisenheimer ${ }^{5}$, and R. I. Davies ${ }^{1}$ \\ ${ }^{1}$ Max-Planck-Institut für extraterrestrische Physik, Giessenbachstrasse, 85748 Garching, \\ Germany \\ Email: schartmann@mpe.mpg.de \\ ${ }^{2}$ Universitätssternwarte München, Scheinerstrasse 1, 81679 München, Germany \\ ${ }^{3}$ Max-Planck-Fellow \\ ${ }^{4}$ ZAH - Landessternwarte Heidelberg, Königstuhl 12, 69117 Heidelberg, Germany \\ ${ }^{5}$ Max-Planck-Institut für Astronomie, Königstuhl 17, 69117 Heidelberg, Germany
}

\begin{abstract}
Recently, high-resolution observations made with the help of the near-infrared adaptive optics integral field spectrograph SINFONI at the VLT proved the existence of massive and young nuclear star clusters in the centers of a sample of Seyfert galaxies. With the help of high-resolution hydrodynamical simulations with the PLUTO code, we follow the evolution of such clusters, especially focusing on mass and energy feedback from young stars. This leads to a filamentary inflow of gas on large scales (tens of parsecs), whereas a turbulent and very dense disk builds up on the parsec scale. Here we concentrate on the long-term evolution of the nuclear disk in NGC 1068 with the help of an effective viscous disk model, using the mass input from the large-scale simulations and accounting for star formation in the disk. This two-stage modeling enables us to connect the tens-of-parsecs scale region (observable with SINFONI) with the parsec-scale environment (MIDI observations). At the current age of the nuclear star cluster, our simulations predict disk sizes of the order 0.8 to $0.9 \mathrm{pc}$, gas masses of order $10^{6} M_{\odot}$, and mass transfer rates through the inner boundary of order $0.025 M_{\odot} \mathrm{yr}^{-1}$, in good agreement with values derived from observations.
\end{abstract}

Keywords. galaxies: Seyfert, galaxies: nuclei, galaxies: ISM, galaxies: individual (NGC 1068), hydrodynamics, methods: numerical, ISM: evolution, ISM: kinematics and dynamics, black hole physics, stars: mass loss

\section{Introduction}

Nuclear activity is an important phase in the evolution of galaxies. Whenever enough gas is fed onto the central supermassive black holes, galaxies become "active" and their nuclear region lights up significantly, compared to non-active phases of their evolution. The infalling material builds up a rapidly rotating accretion disk, which heats up viscously. The emerging UV/optical radiation illuminates a gas and dust reservoir — the so-called "molecular torus." Given the high opacity of the dust in this wavelength range, the torus morphology is able to geometrically unify two observed classes of active galaxies: type 1 objects, where the torus is viewed face-on (and all of the characteristics of the central region are visible) and type 2 objects (torus viewed edge-on), where most of the light from the accretion disk is absorbed and re-emitted in the infrared regime. This is the essence of the "unified scheme" of AGN. Despite its general success when probed with observational data, the nature of the obscuring gas and dust reservoir the torus, as well as the triggering mechanism of such phases - is still a matter of active research. To assess the final stages of mass transport through the central tens-of-parsecs 
region, nearby objects are needed, due to the limitations on resolution posed by currently available instruments. Therefore, Seyfert galaxies are an ideal testbed to study these processes. MIDI (MID-infrared Interferometer) observations recently resolved the innermost parts of the torus directly for the first time (e.g., Jaffe et al. 2004; Tristram et al. 2007, 2009; Burtscher et al. 2009) and find evidence for a clumpy dust distribution. In these proceedings, we numerically model a scenario that is able to describe the build-up and evolution of a nuclear gas disk or torus. Our simulations are put into context by Davies et al. (these proceedings).

\section{Three-Dimensional Hydrodynamical Simulations}

Recently, high-resolution observations with the help of the near-infrared adaptive optics integral field spectrograph SINFONI at the VLT proved the existence of massive and young nuclear star clusters in the centers of a sample of Seyfert galaxies (Davies et al. 2007 and these proceedings). With the help of three-dimensional high-resolution hydrodynamical simulations using the PLUTO code, we follow the evolution of such clusters. The gas ejection from their stars provide both material for the obscuration within the mentioned unified scheme of AGN and a reservoir to fuel the central, active region, and it additionally drives turbulence in the interstellar medium on tens-of-parsec scales. We start our simulations after the very violent phase of supernova type II explosions and fast winds. In order to enable a direct data comparison, we constrain our input parameters with observed values of the well-studied nearby Seyfert galaxy NGC 1068. A summary of the parameters used for the simulations shown in these proceedings is given in Table 1. Approximately 50 Myrs after the starburst, the mass loss of the newly born stellar popu-

Table 1. Parameters of Our Standard Models

\begin{tabular}{|c|c|c|c|c|}
\hline \multicolumn{3}{|c|}{ Hydrodynamical model } & \multicolumn{2}{|c|}{ Effective disk model } \\
\hline Parameter & Value & Reference & Parameter & Value \\
\hline$M_{\mathrm{BH}}$ & $8 \times 10^{6} M_{\odot}$ & L03 & $R_{\text {in }}$ & $0.1 \mathrm{pc}$ \\
\hline$M_{*}$ & $2.2 \times 10^{8} M_{\odot}$ & D07 & $R_{\text {out }}$ & $100.0 \mathrm{pc}$ \\
\hline$M_{\text {gas }}^{\text {ini }}$ & $1.0 \times 10^{2} M_{\odot}$ & & $\delta$ & 0.2 \\
\hline$R_{\mathrm{c}}^{\mathrm{s}}$ & $25 \mathrm{pc}$ & G03 & $T$ & $400 \mathrm{~K}$ \\
\hline$R_{\mathrm{T}}$ & $5 \mathrm{pc}$ & & $\alpha_{\nu}$ & 0.05 \\
\hline$R_{\text {in }}$ & $0.2 \mathrm{pc}$ & & $t_{\text {cluster }}^{\text {start }}$ & $50 \mathrm{Myr}$ \\
\hline$R_{\text {out }}$ & $50 \mathrm{pc}$ & & $t_{\text {cluster }}^{\text {end }}$ & $300 \mathrm{Myr}$ \\
\hline$\sigma_{*}$ & $100 \mathrm{~km} \mathrm{~s}^{-1}$ & D07 & $n_{\mathrm{r}}$ & 5000 \\
\hline$\beta$ & 0.5 & & & \\
\hline$T_{\text {ini }}$ & $2.0 \times 10^{6} \mathrm{~K}$ & & & \\
\hline$\dot{M}_{\mathrm{n}}$ & $9.1 \times 10^{-10} M_{\odot} \mathrm{yr}^{-1}$ & J01 & & \\
\hline$M_{\mathrm{PN}}$ & $0.5 M_{\odot}$ & & & \\
\hline$\Gamma$ & $5 / 3$ & & & \\
\hline
\end{tabular}

Mass of the black hole $\left(M_{\mathrm{BH}}\right)$, normalization constant of the stellar potential $\left(M_{*}\right)$, initial gas mass $\left(M_{\mathrm{gas}}^{\mathrm{ini}}\right)$, cluster core radius $\left(R_{\mathrm{c}}\right)$, torus radius $\left(R_{\mathrm{T}}\right)$, inner radius $\left(R_{\mathrm{in}}\right)$, outer radius $\left(R_{\mathrm{out}}\right)$, stellar velocity dispersion $\left(\sigma_{*}\right)$, exponent of the angular momentum distribution of the stars $(\beta)$, initial gas temperature $\left(T_{\mathrm{ini}}\right)$, normalised mass injection rate $\left(\dot{M}_{\mathrm{n}}\right)$, mass of a single injection $\left(M_{\mathrm{PN}}\right)$ and adiabatic exponent $(\Gamma)$, thickness of the disk $(\delta=$ disk scale height / radius of the disk), gas temperature $(T)$, alpha viscosity parameter $\left(\alpha_{\nu}\right)$, age of the nuclear star cluster at the beginning of the simulations $\left(t_{\text {cluster }}^{\text {start }}\right)$ and at the end $\left(t_{\text {cluster }}^{\text {end }}\right)$ and resolution of the simulation $\left(n_{\mathrm{r}}\right)$. The references are: L03 (Lodato \& Bertin 2003); D07 (Davies et al. 2007); G03 (Gallimore \& Mathews 2003); and J01 (Jungwiert et al. 2001). 

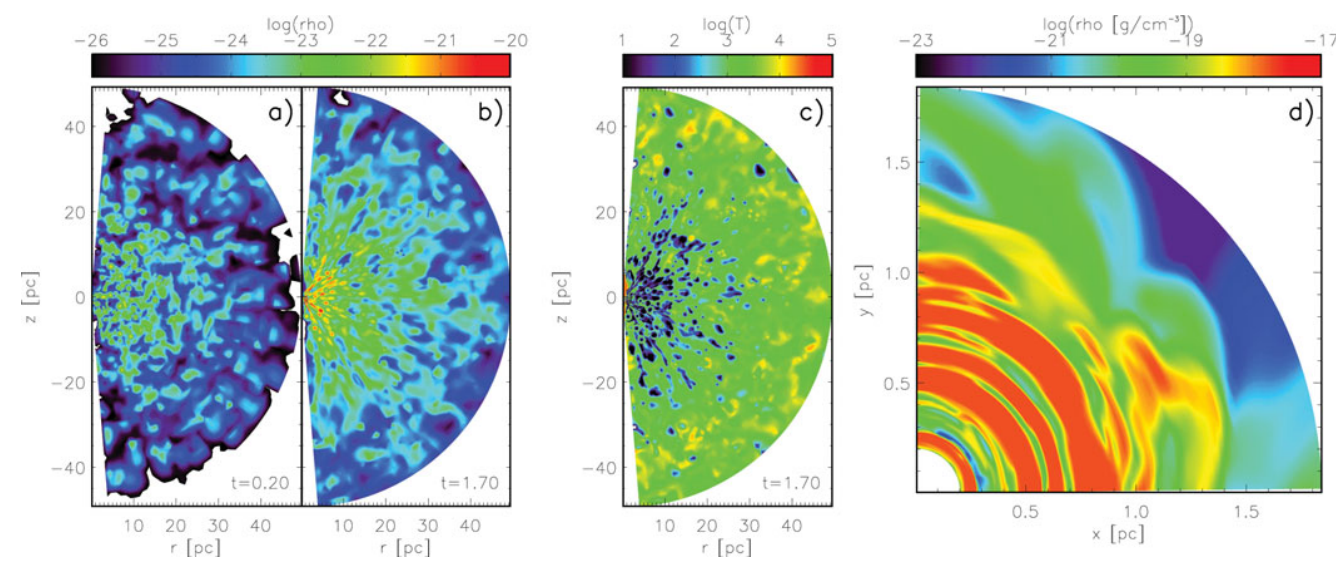

Figure 1. Snapshots of the density distribution in a meridianal plane of our 3D hydrodynamical standard model after (a) 0.2 orbits (corresponding to $7 \times 10^{4} \mathrm{yr}$ ) and (b) 1.7 orbits (approximately $6 \times 10^{5} \mathrm{yr}$ ). Panel (c) shows the temperature distribution in the same meridianal plane after 1.7 orbits. Panel (d) is zoomed into the innermost part, showing the nuclear disk component.

lation is dominated by slow winds and the ejection of planetary nebulae. Then the input of energy into the ambient medium is low enough to enable transport of gas towards the center, allowing for the triggering of activity there (see also Davies et al., these proceedings). A similar time delay between the nuclear starburst and the onset of nuclear activity has also been found observationally, leading to the same conclusions as our work (Davies et al. 2007). We model the mass-loss of the stellar population as the ejection of single expanding clumps of gas, to which we give the velocity of the underlying stellar distribution at the point of emission and that is made up of a rotation component and a random component $\dagger$. These blobs of gas - confined by cooling instability - merge to form larger entities, thereby dissipating a fraction of their initial turbulent motions, and get transported towards the center, forming slightly elongated filaments. These processes lead to a vertically wide, clumpy or filamentary distribution of inflowing gas on tens-ofparsec scales (see Figure 1a,b,c), whereas a turbulent and very dense disk builds up on the parsec scale (see Figure 1d). A similar two-component structure has been found in interferometric observations from the MIDI instrument of NGC 1068 (Raban et al. 2009) and the Circinus galaxy (Tristram et al. 2007).

As these are computationally very expensive simulations and, as we currently are unable to take all physical processes occurring in the innermost few parsecs into account in the three-dimensional hydrodynamical calculations, we treat the inner disk separately with the help of an effective disk model, as described in $\S 3$.

\section{Effective Disk Modeling}

In order to capture the relevant physics in the inner region, we treat this disk separately in a one-dimensional, axisymmetric simulation that also enables a direct comparison with observations. Due to the generally unknown physical mechanism responsible for tranferring angular momentum and mass in the disk, we concentrate on the effects of a parameterized turbulent viscosity and additionally take star formation into account. Following Pringle (1981) and Lin \& Pringle (1987), the viscous evolution of such a disk

$\dagger$ These nuclear star clusters possess a significant velocity dispersion and rotate with sub-Keplerian velocity. 
can be described by

$$
\frac{\partial}{\partial t} \Sigma(t, R)+\frac{1}{R} \frac{\partial}{\partial R}\left\{\left[\frac{\partial}{\partial R}\left(\nu_{\alpha} \Sigma(t, R) R^{3} \frac{d \Omega(R)}{d R}\right)\right]\left[\frac{d\left(R^{2} \Omega\right)}{d R}\right]^{-1}\right\}=S,
$$

where $\Sigma$ is the gas surface density within the disk, $R$ is the radial distance from the center, $t$ the time, $\nu_{\alpha}$ the assumed alpha viscosity, $\Omega$ the Keplerian rotation frequency, and $S$ includes a source term for the mass input from our three-dimensional hydrodynamical simulations $(\S 2)$ and a sink term, taking star formation into account with the help of the Kennicutt-Schmidt law. The infalling material is placed according to its angular momentum in a Keplerian rotating disk structure (Figure 2a). Gas consumed by star formation is removed from the simulations and will no longer participate in the dynamical evolution. We solve equation (3.1) numerically with the people solver in MATLAB $\dagger$. This two-stage modeling process enables us to directly compare our results to observed properties.

\section{Results and Data Comparison}

Figure $2 \mathrm{~b}$ shows the comparison of the mass contributions of the various components from the 1D modeling for an assumed alpha viscosity parameter of $\alpha=0.05$ and a gas temperature of $400 \mathrm{~K}$. The input parameters of the simulations have been constrained by observations of the nearby Seyfert 2 galaxy NGC 1068 (see Table 1). At the current age of the starburst in NGC 1068 of approximately $250 \mathrm{Myr}$, the largest fraction of the integrated gas mass introduced into the disk simulations (given by the dashed line in Figure $2 \mathrm{~b}$ ) has been accreted with the help of viscous processes (dash-dotted line). A slightly smaller amount has been transformed into stars, and a few times $10^{6} M_{\odot}$ of gas remains in the disk (solid line), which has been built up within a few million years. The latter compares well with the disk mass Kumar (1999) finds by fitting a clumpy

\section{$\dagger$ http://www.mathworks.com/products/matlab/}
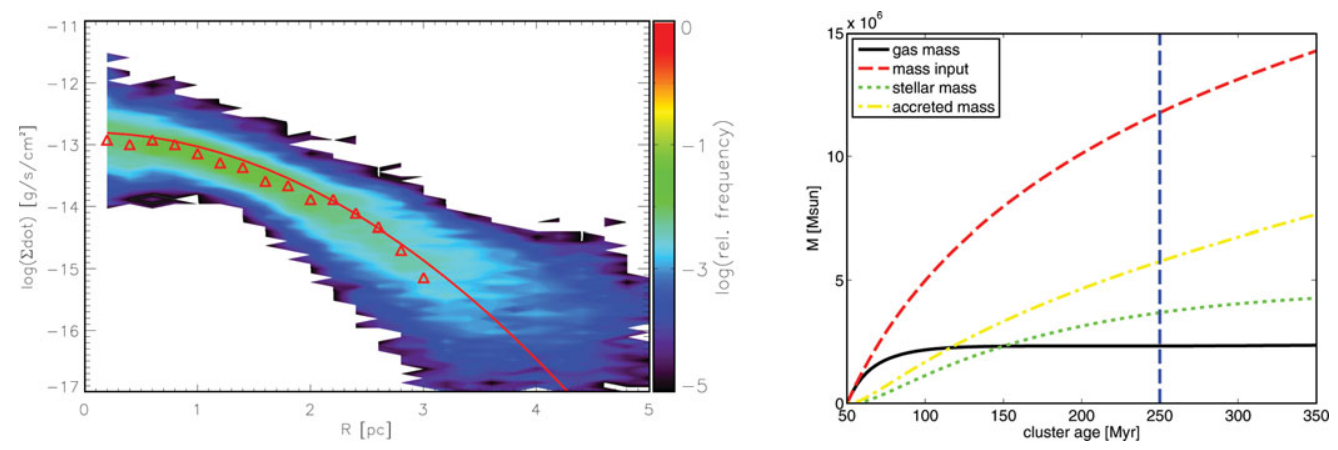

Figure 2. Left: Mass input into our effective disk model, shown as the growth of the gas surface density at different radii, flowing in through a sphere with radius of $2.5 \mathrm{pc}$ from our 3D PLUTO turbulent torus simulation. The input radii are set according to an angular momentum distribution of a Keplerian disk. Shown is the logarithm of a histogram for 100 timesteps with a resolution of approximately $3400 \mathrm{yr}$ between an evolution time of $0.7 \mathrm{Myr}$ and $1.0 \mathrm{Myr}$, where the $3 \mathrm{D}$ hydro simulation is already in an equilibrium state. The triangles denote the maxima of each radial bin, the parabola is the parameterization used as source term in the effective disk simulations. Right: Comparison of mass contributions of the various components of our 1D effective disk model for an alpha viscosity parameter of 0.05 and a gas temperature of $400 \mathrm{~K}$. The vertical dashed line denotes the estimated current age of the nuclear star cluster in NGC 1068 . 
disk model to the maser detections. Figure 3a shows the surface density distribution for three simulations with varying strengths of the viscosity: $\alpha=0.05,0.1$ and 0.2 . For comparison, the range of slopes of the density distribution as inferred from MIDI observations of a sample of nearby Seyfert galaxies is overplotted as dashed line segments. The sizes of the corresponding dust distributions we derive from these curves amount to 0.8 to $0.9 \mathrm{pc}$, which is remarkably similar to values obtained with the MIDI instrument at approximately $0.7 \mathrm{pc}$ and at the estimated extent of the maser disk ( 0.65 to $1.1 \mathrm{pc})$. Finally, the total accretion rate through the inner boundary of our disk simulations is plotted in Figure 3b for the $\alpha$ parameter study discussed above. Larger values of $\alpha$ clearly lead to a larger mass accretion rate, and at the current age of the nuclear stellar cluster, the derived values agree well with typical accretion rates of Seyfert galaxies of a few times $10^{-3}$ to $10^{-2} M_{\odot} \mathrm{yr}^{-1}$ (Jogee 2006). However, NGC 1068 seems to be in a heavily accreting state, which can be accomodated in our model only when assuming, for example, clumpy accretion.

\section{Concluding Remarks}

In these proceedings, we show that evolving stars from a massive and young nuclear star cluster, as found in nearby Seyfert galaxies, provide enough gas to assemble a parsecsized nuclear gas disk. To this end, we combine a three-dimensional treatment of the mass-loss from an evolving nuclear star cluster with a simplified model for the innermost parsec scale region, where a nuclear disk builds up. As far as possible, we derive input parameters of our simulations from observations of the nearby and well-studied Seyfert 2 galaxy NGC 1068. This two-stage analysis enables us (i) to do a long term evolution study, (ii) to link the tens of parsec scale region of galactic nuclei (observed with the SINFONI instrument) to sub-parsec scales (probed by MIDI and in water maser emission), and (iii) to test our model directly with a large number of observational results. At the current age of its nuclear starburst of $250 \mathrm{Myr}$, our simulations yield disk sizes of the order 0.8 to $0.9 \mathrm{pc}$, gas masses of order $10^{6} M_{\odot}$, and mass transfer rates of order $0.025 M_{\odot} \mathrm{yr}^{-1}$ through the inner rim of the disk, in good comparison with observed disk and torus properties. On basis of these comparisons, we conclude that the proposed scenario seems to be a reasonable model and shows that nuclear star formation activity and subsequent AGN activity are intimately related.
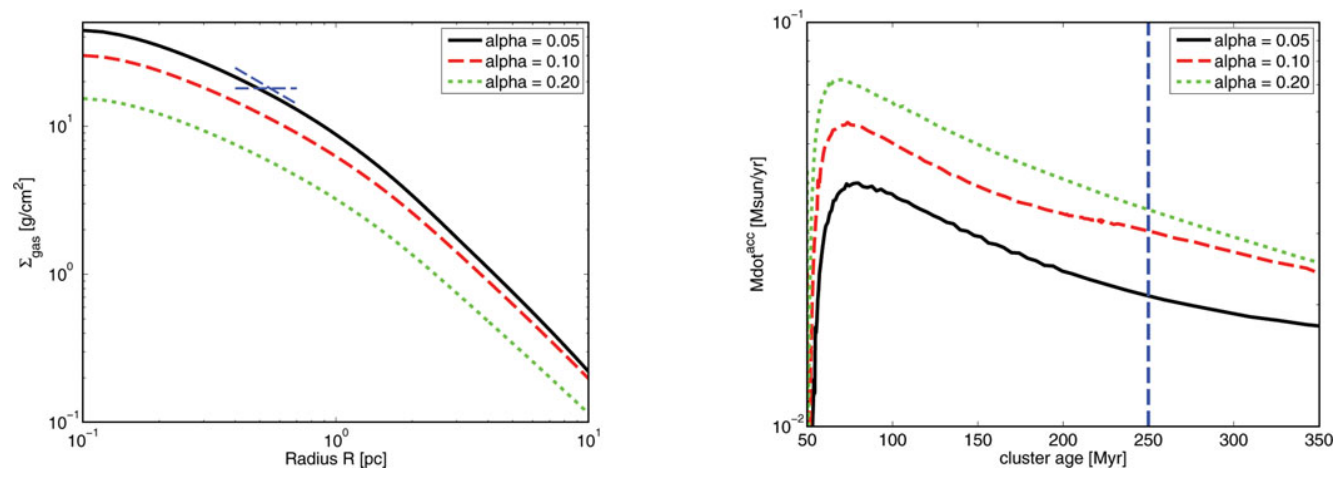

Figure 3. Left: Final gas density distribution at a cluster age of $250 \mathrm{Myr}$. Superposed as dashed lines are the two limiting curves for the range of possible surface density distributions as determined from MIDI observations (Kishimoto et al. 2009). Right: Total accretion rate of gas through the inner boundary of the domain. 


\section{Acknowledgements}

Part of the numerical simulations have been carried out on the SGI Altix 4700 HLRB II of the Leibniz Computing Centre in Munich (Germany).

\section{References}

Burtscher, L., Jaffe, W., Raban, D., Meisenheimer, K., Tristram, K. R. W., \& Röttgering, H. 2009, ApJ, 705, L53

Davies, R. I., Mueller Sánchez, F., Genzel, R., Tacconi, L. J., Hicks, E. K. S., Friedrich, S., \& Sternberg, A. 2007, ApJ, 671, 1388

Gallimore, J. F. \& Matthews, L. 2003, in Active Galactic Nuclei: From Central Engine to Host Galaxy, ed. L. C. Ho \& J.-M. Wang (San Francisco: ASP), p. 501

Jaffe, W., Meisenheimer, K., Röttgering, H. J. A., Leinert, Ch., Richichi, A., Chesneau, O., Fraix-Burnet, D., Glazenborg-Kluttig, A., Granato, G.-L., Graser, U., Heijligers, B., Köhler, R., Malbet, F., Miley, G. K., Paresce, F., Pel, J.-W., Perrin, G., Przygodda, F., Schoeller, M., Sol, H., Waters, L. B. F. M., Weigelt, G., Woillez, J., \& de Zeeuw, P. T. 2004, Nature, 429, 47

Jogee, S. 2006, in Lecture Notes in Physics, 693, 143

Jungwiert, B., Combes, F., \& Palouš, J. 2001, A\&A, 376, 85

Kishimoto, M., Hönig, S. F., Tristram, K. R. W., \& Weigelt, G. 2009, A\&A, 493, L57

Kumar, P. 1999, ApJ, 519, 599

Lin, D. N. C. \& Pringle, J. E. 1987, ApJ, 320, L87

Lodato, G. \& Bertin, G. 2003, A\&A A, 398, 517

Pringle, J. E. 1981, ARAA, 19, 137

Raban, D., Jaffe, W., Röttgering, H., Meisenheimer, K., \& Tristram, K. R. W. 2009, MNRAS, 394,1325

Schartmann, M., Meisenheimer, K., Klahr, H., Camenzind, M., Wolf, S., \& Henning, Th. 2009, MNRAS, 393, 759

Tristram, K. R. W., Meisenheimer, K., Jaffe, W., Schartmann, M., Rix, H.-W., Leinert, Ch., Morel, S., Wittkowski, M., Röttgering, H., Perrin, G., Lopez, B., Raban, D., Cotton, W. D., Graser, U., Paresce, F., \& Henning, Th. 2007, A\&\&A, 474, 837

Tristram, K. R. W., Raban, D., Meisenheimer, K., Jaffe, W., Röttgering, H., Burtscher, L., Cotton, W. D., Graser, U., Henning, Th., Leinert, Ch., Lopez, B., Morel, S., Perrin, G., \& Wittkowski, M. 2009, A\&BA, 502, 67 